\title{
Thrombotic circuit complications during venovenous extracorporeal membrane oxygenation in COVID-19
}

\author{
Xavier Bemtgen ${ }^{1,2}$ (I) Viviane Zotzmann ${ }^{1,2} \cdot$ Christoph Benk $^{3} \cdot$ Jonathan Rilinger ${ }^{1,2} \cdot$ Katrin Steiner $^{1,2,4}$. \\ Alexander Asmussen ${ }^{1,2} \cdot$ Christoph Bode $^{1,2} \cdot$ Tobias Wengenmayer $^{1,2} \cdot$ Sven Maier $^{3} \cdot$ Dawid L. Staudacher $^{1,2}$
}

Published online: 11 July 2020

(c) The Author(s) 2020

\begin{abstract}
The novel coronavirus SARS-CoV-2 and the resulting disease COVID-19 causes pulmonary failure including severe courses requiring venovenous extracorporeal membrane oxygenation (V-V ECMO). Coagulopathy is a known complication of COVID-19 leading to thrombotic events including pulmonary embolism. It is unclear if the coagulopathy also increases thrombotic circuit complications of the ECMO. Aim of the present study therefor was to investigate the rate of V-V ECMO complications in COVID-19. We conducted a retrospective registry study including all patients on V-V ECMO treated at our centre between 01/2018 and 04/2020. COVID-19 cases were compared non- COVID-19 cases. All circuit related complications resulting in partial or complete exchange of the extracorporeal system were registered. In total, 66 patients were analysed of which $11(16.7 \%)$ were SARS-CoV-2 positive. The two groups did not differ in clinical parameters including age (COVID-19 59.4 vs. non-COVID-19 58.1 years), gender (36.4\% vs. 40\%), BMI (27.8 vs. 24.2) and severity of illness as quantified by the RESP Score (1pt. vs 1 pt.). 28 days survival was similar in both groups $(72.7 \%$ vs. $58.2 \%)$. While anticoagulation was similar in both groups $(p=0.09)$, centrifugal pump head thrombosis was more frequent in COVID-19 $(9 / 11$ versus $16 / 55 p<0.01)$. Neither the time to first exchange $(p=0.61)$ nor blood flow at exchange $(p=0.68)$ did differ in both groups. D-dimer levels prior to the thrombotic events were significantly higher in COVID-19 (mean 15.48 vs 26.59 , $p=0.01$ ). The SARS-CoV-2 induced infection is associated with higher rates of thrombotic events of the extracorporeal system during V-V ECMO therapy.
\end{abstract}

Keyword V-V ECMO · COVID-19 · Thrombotic complications · Pump head thrombosis

Sven Maier and Dawid L. Staudacher have equal contribution.

Xavier Bemtgen

Xavier.bemtgen@uniklinik-freiburg.de

1 Department of Medicine III (Interdisciplinary Medical Intensive Care), Medical Center-University of Freiburg, Faculty of Medicine, University of Freiburg, Hugstetter Straße 55, 79106 Freiburg, Germany

2 Department of Cardiology and Angiology I, Heart Center Freiburg University, Faculty of Medicine, University of Freiburg, Freiburg, Germany

3 Department of Cardiovascular Surgery, Heart Center Freiburg University, Faculty of Medicine, University of Freiburg, Freiburg, Germany

4 Department of Anaesthesia, Intensive Care Medicine and Pain Medicine, Medical University of Vienna, Vienna, Austria

\author{
Abbreviations \\ aPTT Activated partial thromboplastin time \\ ARDS Acute respiratory distress syndrome \\ COVID-19 Coronavirus disease 2019 \\ ICU Intensive care unit \\ SARS-CoV-2 Severe acute respiratory syndrome corona- \\ virus 2 \\ V-V ECMO Venovenous extracorporeal membrane \\ oxygenation
}

\section{Highlights}

- This study compared the rate of thrombotic circuit complications in COVID-19 V-V ECMO patients to historical controls.

- The two groups were comparable. 
- Patients with COVID-19 had significantly more thrombotic circuit complications and D-Dimer levels prior to exchange were significantly higher.

\section{Introduction}

The novel severe acute respiratory syndrome coronavirus 2 (SARS-CoV-2) and the related coronavirus disease 2019 (COVID-19) has grown to a worldwide pandemic. Patients infected with SARS-CoV-2 have a great variety of symptoms reaching from asymptomatic course to sever pulmonary impairment [1]. In one epidemiological study reporting 1.099 patients, $15.7 \%$ were categorized as "sever" on admission and 37 developed an pulmonary failure comparable to an acute respiratory distress syndrome (ARDS) as proposed by Archer et al.[2, 3]. The mortality seems to be considerable in critically ill patients admitted to the intensive care unit (ICU) as one early study reported a mortality of $61.5 \%$ at 28 days [4]. Here, $67 \%$ developed an ARDS-like phenotype during the infection with SARS-CoV-2 [4].

Treatment of acute respiratory failure of a COVID-19 infection consists of different pulmonary support strategies: non-invasive ventilation, invasive ventilation, prone position and in selective cases venovenous extracorporeal membrane oxygenation (V-V ECMO) [5]. Initial reports on V-V ECMO utilization during COVID-19 respiratory failure have described a hospital mortality of 50\% though newer register data reported by the European Extracorporeal Life Support Organization (EuroELSO) seems to be more encouraging with a reported death rate of $17.1 \%$ with a great variety between centers $[6,7]$.

Although scarcity of resources during a pandemic is a major risk, V-V ECMO is still considered a viable option in selective cases following failure of conventional therapy [5].

Newly emerging data on COVID-19 appears to be associated with a high rate of venous and arterial thromboembolic complications with reported cumulative rates of $21 \%$ [8]. To this date, only very limited data is available concerning rate of V-V ECMO complications in this specific patient group. In a large French prospective cohort study, 12 patients with V-V ECMO were reported and a total of three thrombotic occlusions of the centrifugal pump in two patients occurred (8\%) [9].

Aim of the present study therefor was to investigate the rate of V-V ECMO complications in COVID-19 infection in comparison to a retrospective control group from 2018 to 2019 .

\section{Methods}

Data derives from a registry of all patients on V-V ECMO treated at a medical intensive care unit located at a tertiary university hospital. We offer a 24/7 service for referral of ARDS and ECMO patients. As for local policy, decision to cannulate for V-V ECMO is made after multidisciplinary discussion at the bedside.

\section{ECMO circuit}

Two different pump types were used for ECMO: Stöckert Centrifugal Pump Console (SCPC) (LivaNova, Munich, Germany) and the Cardiohelp-System (Maquet, Rastatt, Germany). For interhospital transportation of patients after ECMO implantation the Lifebox console (LivaNova, Munich, Germany) or the Cardiohelp-System was used. The tubing sets and Oxygenators consists of three sets: HLS 7.0Set (Priming volume: $273 \mathrm{ml}$, Surface: $1.8 \mathrm{~m}^{2}$ ) (Maquet, Rastatt, Germany) and two customized tubing sets with a LivaNova EOS ECMO Oxygenator (Priming volume: $150 \mathrm{ml}$, Surface: $1.2 \mathrm{~m}^{2}$ ) or EuroSets A.L.ONE ECMO Oxygenator (Priming volume: $225 \mathrm{ml}$, Surface: $1.8 \mathrm{~m}^{2}$ ) (EuroSets, Medolla, Italy). If two oxygenators were used in parallel, Y-connectors were used before and after the oxygenators to split the blood flow. All Sets were fully coated (Maquet: Bioline, LivaNova and EuroSets: Phosphorylcholine). The Priming consists of $700 \mathrm{ml}$ electrolyte solution and $5000 \mathrm{IE}$ unfractionated heparin. In case of heparin-induced thrombocytopenia, no unfractionated heparin was used for priming.

\section{V-V ECMO management}

All ECMO circuits were checked once a day by a perfusionist and three times a day by each a nurse and a physician for visible thrombus formations, lipid deposits, changes of the pump head bearing. Indications for exchange of the whole ECMO-System, except the cannulas, were thrombus formations with the risk of thromboembolic events or system failure. In case of visible thrombus formation at the pump head or running noise an isolated change of the pump head was performed if gas exchange was sufficient and no further thrombus formation in the ECMO circuit was visible. Isolated change of connectors in the tubing set was necessary in case of thrombus formation at a connector with no thrombus at the pump head or oxygenator. All exchanges were carried out jointly by a registered nurse, a perfusionist and an ECMO specialist.

As for this research, only changes in the ECMO circuits requiring an exchange of the pump head or the whole system were considered of clinical importance. Each exchange was judged by a perfusionist and an ECMO physician 
independently reviewing all available documentation. All non-thrombotic complications requiring exchange were excluded. In case of dissent of the two independent reviewers, the exchange was included in the analysis.

\section{Anticoagulation}

During V-V ECMO therapy, anticoagulation with continuous infusion of unfractionated heparin was administered as defined by standard operating procedure. When there was no other indication for therapeutic anticoagulation, the target range for activated partial thromboplastin time (aPTT) was 40-50 s and adjusted depending on thrombus burden or bleeding events. If there was suspicion or proof of heparin-induced thrombocytopenia or insufficient thrombus control in the circuit with unfractionated heparin, the anticoagulation was switched to argatroban with an aPTT target of $>60 \mathrm{~s}$. As reports of thrombotic complications in COVID-19 emerged, anticoagulation target was increased from aPTT 40-50 s to aPTT 50-70 s.

\section{Data and ethics}

COVID-19 patients were recruited prospectively as was data acquisition. The control group consisted of all V-V ECMO patients treated at our hospital during the years 2018 to 2019 and were recruited retrospectively as was data acquisition. This research was covered by an ethics agreement (ethics committee of the Albert-Ludwigs-University, Freiburg, Germany; file numbers 151/14 and 234-20). Consent was not required as approved by the ethics committee. This study was performed in line with the principles of the 1964 Declaration of Helsinki and its later amendments. Demographic data (age, sex and body dimensions), baseline characteristics related to the V-V ECMO (duration, indication, cannula size), anticoagulation strategy, D-dimers measurements, survival to discharge and presence of complications related to the V-V ECMO device were collected.

\section{Statistical analysis}

For data analysis, SPSS (version 23, IBM Statistics) or Prism (version 8, GraphPad) were employed. For statistical analysis, unpaired $t$-test, Fisher's-exact/chi-square test, and Log-rank/Gehan Breslow test were used as applicable and a $p$ value of $<0.05$ was considered statistically significant. All data are presented as absolute number (percent of all patients) or as median with inter quartile range if not stated otherwise.

\section{Results}

A total of $66 \mathrm{~V}-\mathrm{V}$ ECMO patients were included, eleven patients who were tested positive for SARS-CoV-2 and 55 patients treated at our hospital during the years 2018 and 2019. 28 days survival was $72.7 \%$ in the COVID-19 group and $58.2 \%$ for the non-COVID-19 group. At the time of conception of the manuscript two COVID-19 patients were still in ongoing V-V ECMO therapy. The median age was 58.4 (46.9-66.2) years at time of V-V ECMO implantation and the group consisted of 40 men and 26 female (39.4\%). Age, sex, body measurements and severity of illness were similar between both groups. Primarily SCPC was used as V-V ECMO system and only in 18\% (COVID-19-group) and 13\% (non-COVID-19-group) of the cases a Cardiohelp-System.

Table1 Baseline characteristics

\begin{tabular}{|c|c|c|c|c|}
\hline & All & Covid19 & Non-Covid19 & $\mathrm{p}$ value \\
\hline Female & $26 / 66(39.39 \%)$ & $4 / 11(36.36 \%)$ & $22 / 55(40 \%)$ & 1.000 \\
\hline 28 days survival & $40 / 66(60.61 \%)$ & $8 / 11(72.73 \%)$ & $32 / 55(58.18 \%)$ & 0.505 \\
\hline Duration of ICU stay [days] & $16.73(9.36-29.90)$ & $27.89(11.98-33.23)$ & $14.71(9.15-24.76)$ & 0.404 \\
\hline Duration of V-V ECMO [days] & $7.82(4.41-17.97)$ & $17.94(7.8-23.75)$ & $7.49(4.15-16.34)$ & 0.242 \\
\hline $\begin{array}{l}\text { Indication for anticoagulation prior to } \\
\text { V-V ECMO }\end{array}$ & $10 / 66(15.15 \%)$ & $1 / 11(9.09 \%)$ & $9 / 55(16.36 \%)$ & 1.000 \\
\hline Height $[\mathrm{cm}]$ & $174.5(168.8-180)$ & $175(170-180)$ & $173(165-180)$ & 0.430 \\
\hline Body weight $[\mathrm{kg}]$ & $80(70.23-90)$ & 85 (79-95) & $77.50(70-90)$ & 0.621 \\
\hline Body-Mass-Index & $24.69(23.51-31.05)$ & $27.78(25.06-33.87)$ & $24.22(23.49-30.86)$ & 0.8450 \\
\hline Age [years] & $58.36(46.85-66.23)$ & $59.38(49.81-61.05)$ & $58.12(44.35-66.51)$ & 0.808 \\
\hline APACHE II & $23.5(18.75-30)$ & $29(27-30)$ & $22(18-31)$ & 0.104 \\
\hline SOFA & $12(10-14.25)$ & $14(13-16)$ & $12(9-14)$ & 0.015 \\
\hline RESP Score & $1((-1)-3)$ & $1(0-2)$ & $1((-2)-4)$ & 0.845 \\
\hline
\end{tabular}

APACHE II acute physiology and chronic health score, RESP Score respiratory extracorporeal membrane oxygenation survival prediction score, SOFA sepsis-related organ failure assessment score 
Table2 V-V ECMO related complications and circuit related characteristics as well as laboratory findings at time exchange

\begin{tabular}{|c|c|c|c|c|}
\hline & All & Covid19 & Non-Covid19 & $\mathrm{p}$ value \\
\hline Total V-V ECMO time (days) & 916.9 & 206.1 & 710.8 & \\
\hline Number of patients with exchange & $22(33.33 \%)$ & $7(63.64 \%)$ & $15(18.18 \%)$ & 0.033 \\
\hline Total number of pump changes & 25 & 9 & 16 & \\
\hline Total number of system changes & 13 & 4 & 9 & \\
\hline Excluded & 10 & none & 10 & \\
\hline Exchanges/week of therapy & 0.29 & 0.44 & 0.25 & \\
\hline Flow prior to exchange & $2.82(2.00-3.95)$ & $2.44(2.01-4.64)$ & $3.00(1.88-3.68)$ & 0.684 \\
\hline D-Dimers before change & $19.42(9.94-35.2)$ & $35.2(16.53-35.2)$ & $15.8(7.52-19.62)$ & 0.005 \\
\hline D-Dimers after change & $11.44(8.57-21.65)$ & $12.78(9.39-35.2)$ & $10.0(6.95-16.41)$ & 0.160 \\
\hline Runtime to first change & $7.04(3.39-10.96)$ & $7.9(5.81-16.77)$ & $5.91(3-9.55)$ & 0.612 \\
\hline \multicolumn{5}{|l|}{ Anticoagulatory target } \\
\hline Low aPTT target $40-50 \mathrm{~s}$ & $10(26.32 \%)$ & $0(0 \%)$ & $10(40 \%)$ & 0.008 \\
\hline Medium aPTT target 50-60 s & $8(21.05 \%)$ & $3(23.08 \%)$ & $5(20 \%)$ & 1.000 \\
\hline High aPTT target $>60 \mathrm{~s}$ & $9(23.68 \%)$ & $2(15.38 \%)$ & $7(28 \%)$ & 0.456 \\
\hline Argatroban $($ aPTT target $>60 \mathrm{~s})$ & $11(28.95 \%)$ & $8(61.54 \%)$ & $3(12 \%)$ & 0.003 \\
\hline
\end{tabular}

$V$-V ECMO veno-venous extracorporeal membrane oxygenation, aPTT activated partial thromboplastin time

All V-V ECMO were set up by percutaneous cannulation and mean V-V ECMO duration was 7.82 (4.41-17.97) days, see Table 1.

In total 25 pump head thrombosis requiring exchange in 16 different patients could be registered, nine exchanges for five patients in the COVID-19-group and 16 exchanges for eleven patients in the non-COVID-19 group. Additionally, a total of 13 system exchanges due to thrombus formation were recorded with four in the COVID-19-group and nine in the non-COVID-19 group respectively, see Table 2. The COVID-19-group had a significantly higher number of thrombus formation in the pump head with exchange $(p<0.01)$ and the duration until $50 \%$ of patients had a pump head thrombosis was significantly shorter in COVID-19 patients compared to controls (169h versus $725 \mathrm{~h}, p<0.01$ ), see Fig. 1.

All exchanges occurred during a still sufficiently running $\mathrm{V}-\mathrm{V}$ ECMO circuit with a median blood flow of $2.9 \mathrm{l} / \mathrm{min}$ (3.1 1/min for COVID-19 vs. $2.9 \mathrm{l} / \mathrm{min}$ for non-COVID-19). No complete system failure due to fulminant system thrombosis was documented. The system runtime from start of the extracorporeal circulation to the first exchange did not differ between both groups (7.9 (5.81-16.77) days for COVID-19 vs. 5.91 (3-9.55) days for non-COVID-19, $p=0.612$ ).

Apart from system and pump head exchanges, additional exchange of two Y-connector changes and two Cytosorb ${ }^{\circledR}$ adsorbers in the COVID-19 group were performed due to thrombosis at the connectors respectively because of a complete thrombosis of the Cytosorb ${ }^{\circledR}$ circuit. In the nonCOVID-19 group, no Y-connector or Cytosorb® changes because of thrombotic events were documented. Cytosorb® adsorbers are not routinely used in V-V ECMO patients and indication for adsorber was based on a patient-per-patient level.

When comparing the systemic anticoagulation and the targeted aPTT prior to exchange of the centrifugal pump, targeted aPTT trended to higher target values in the COVID19 group $(p=0.09)$. The lower target range from 40-50 s was only present in the non-COVID-19 group, see Fig. 2. Also, measured aPTT levels were significantly higher in the COVID-19 group prior to the exchange (62.0 (51.5-83.5) seconds for COVID-19 vs 55.0 (44.5-60.0) seconds for non-COVID-19) whereas measurements 24 and $48 \mathrm{~h}$ prior to the exchange showed no significant difference between the groups. D-Dimers were not acquired routinely in the non-COVID-19 group, therefore only 22/25 measurements within $24 \mathrm{~h}$ before a change and 18/25 after a change were available. Here, we did see significantly higher D-dimer values in the COVID-19 group prior to change whereas there was no significant difference after change, see Table 2.

\section{Discussion}

In this study, we found a significantly higher rate of thrombotic complications in the ECMO circuit of COVID-19 patients, especially pump head thrombosis, in comparison to non-COVID-19 patients (63.6\% vs. $18.2 \%$ ).

There is only limited data on thrombotic complications in V-V ECMO for both, non-COVID-19 and COVID-19 patients. A retrospective study from 2014 did report an exchange rate due to thrombosis of $27 \%$ runs [10]. Other data suggest pump head thrombosis rate of $9 \%$ in $\mathrm{V}-\mathrm{V}$ 


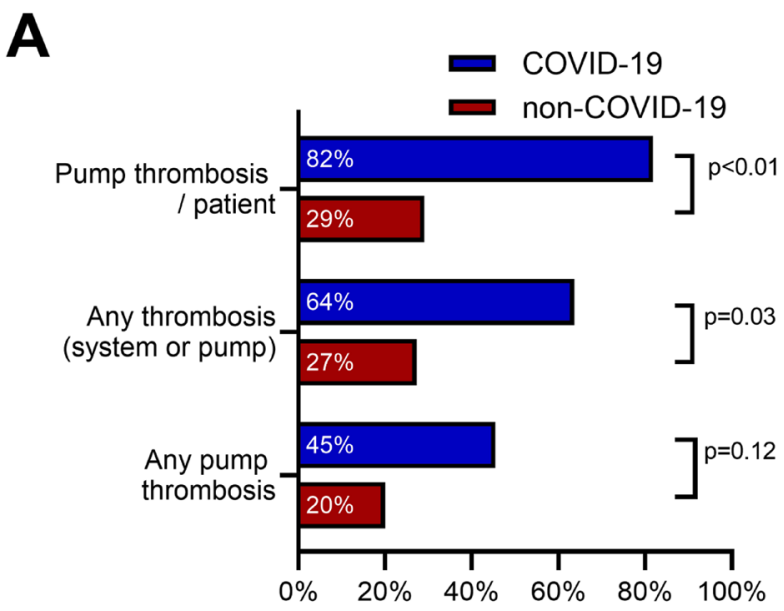

B

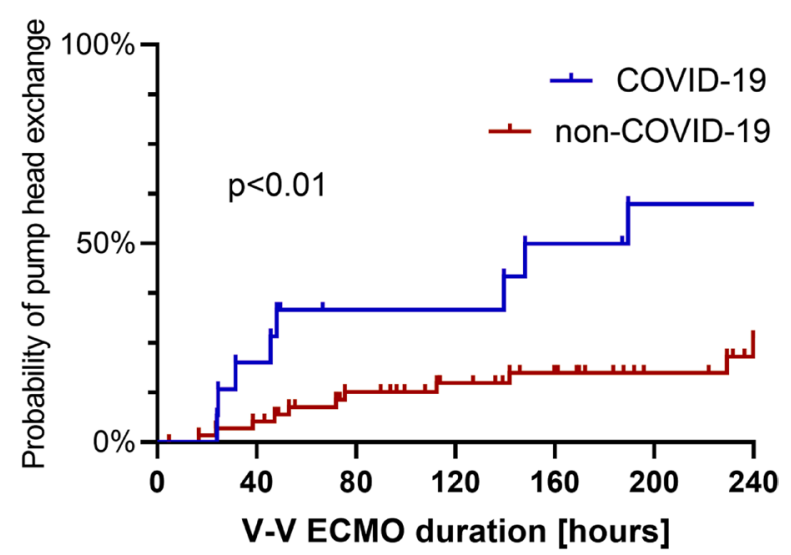

Fig. 1 Thrombotic complications in V-V ECMO circuits. a Number of exchanges due to thrombus formation in different parts of the extracorporeal circuit. b Probability of centrifugal pump exchange due to pump head thrombosis

ECMO [11]. A recent paper on COVID-19 reported three thrombotic occlusions of the centrifugal pump in $2 / 12$ ECMO (25\% of runs) patients [9]. A prospective analyse of used circuits in paediatric patients after removal from extracorporeal support did show a higher overall incidence of thrombus formation in the pump head in centrifugal pumps of $41 \%$ [12]. Therefore, the incidence of pump head thrombosis detected in our non-COVID-19 cohort is in line with literature. The significant higher number of pump head

thrombosis detected in the COVID-19 subgroup is alarming. Thrombus formation in the extracorporeal system, especially the centrifugal pump, if undetected, can result in complete system failure due to complete congestion of the circuit or overheating of the pump shaft.

We noted significantly higher d-dimer values in in COVID-19 patients with thrombus formation in the centrifugal pump compared to control patients. It has been suggested that an incline in d-dimer levels over three days (from 15 to $30 \mathrm{mg} / \mathrm{dL}$ ) correlates highly with occurrence of thrombosis in ARDS patients on V-V ECMO [13]. Although d-dimers are highly elevated in COVID-19, an increase in d-dimers should not only be attributed to the underlying disease but might also indicate pump head thrombosis.

We found no difference in targeted aPTT in our COVID19 and non-COVID-19 group with a trend to higher values in the COVID-19 group but aPTT measured at the day of exchange were significantly higher in the COVID-19 group. Interestingly recent data on thrombotic complications in COVID-19 reported high incidence despite systemic anticoagulation [9]. If higher anticoagulation targets can prevent thrombotic events in COVID-19 and are connected to better outcomes in the whole COVID-19 population (and especially in the V-V ECMO Group) has to be addressed in larger trials.

\section{Limitation}

Being a prospective observational, single-center study, inherent limitations and biases are to be presumed and findings are to be considered hypothesis generating. Data on non-COVID-19 patients was collected retrospectively and therefor incomplete documentation is a limiting factor. Also, primarily SCPC systems are used at our center and therefor no rational subgroup analysis for the different systems could be performed.

\section{Conclusion}

Thrombus formation of the extracorporeal circuit in COVID19 patients is common and potentially life-threatening. Compared to the usual V-V ECMO, the risk is higher and therefor 

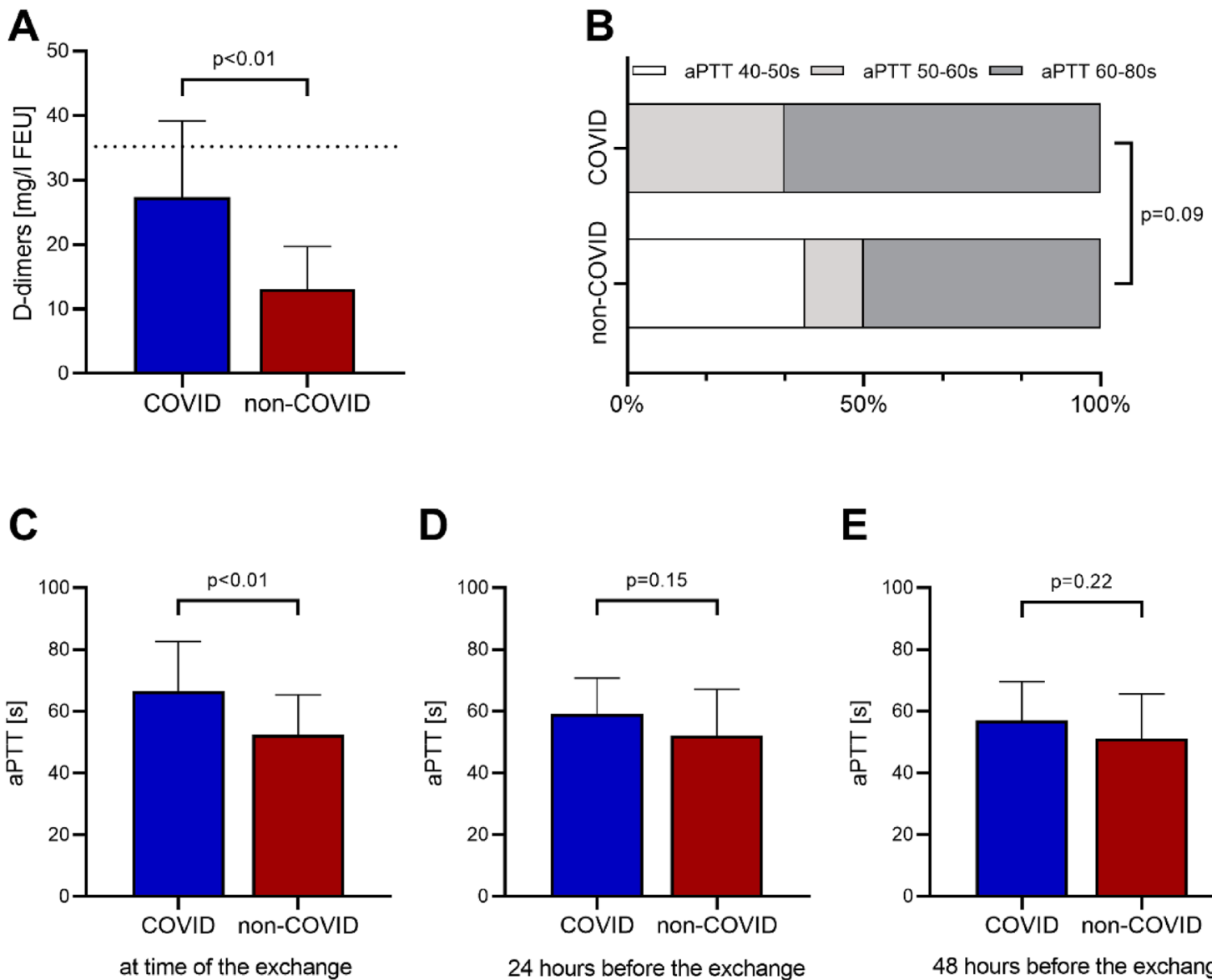

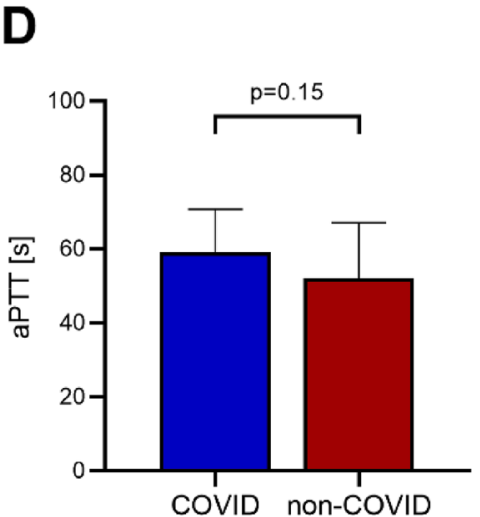

24 hours before the exchange
$\mathbf{E}$

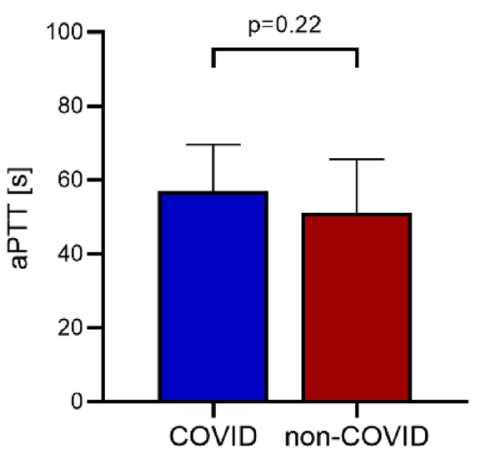

48 hours before the exchange
Fig. 2 Laboratory findings during the exchange of parts or the whole venovenous extracorporeal membrane oxygenation circuit. a D-Dimers levels prior to the exchange, laboratory capped value at $35.2 \mathrm{mg} / \mathrm{l}$. b Anticoagulation target prior to the exchange. c Last

the team should be even more watchful than usual to counteract these kinds of complications.

Acknowledgements Open Access funding provided by Projekt DEAL.

Funding None.

\section{Compliance with ethical standards}

Conflict of interest The authors declare that they have no conflict of interest.

Open Access This article is licensed under a Creative Commons Attribution 4.0 International License, which permits use, sharing, adaptation, distribution and reproduction in any medium or format, as long as you give appropriate credit to the original author(s) and the source, provide a link to the Creative Commons licence, and indicate if changes were made. The images or other third party material in this article are included in the article's Creative Commons licence, unless indicated otherwise in a credit line to the material. If material is not included in the article's Creative Commons licence and your intended use is not permitted by statutory regulation or exceeds the permitted use, you will measured activated partial thromboplastin time (aPTT) prior to the exchange. d aPTT $24 \mathrm{~h}$ prior to the exchange. e aPTT $48 \mathrm{~h}$ prior to the exchange

need to obtain permission directly from the copyright holder. To view a copy of this licence, visit http://creativecommons.org/licenses/by/4.0/.

\section{References}

1. Vetter P, Vu DL, L'Huillier AG et al (2020) Clinical features of covid-19. BMJ. https://doi.org/10.1136/bmj.m1470

2. Guan W, Ni Z, Hu Y et al (2020) Clinical characteristics of coronavirus disease 2019 in China. N Engl J Med 382:1708-1720. https://doi.org/10.1056/NEJMoa2002032

3. Archer SL, Sharp WW, Weir EK. (2020). Differentiating COVID-19 pneumonia from acute respiratory distress syndrome (ARDS) and high altitude pulmonary edema (HAPE): therapeutic implications. Circulation CIRCULATIONAHA.120.047915.

4. Yang X, Yu Y, Xu J et al (2020) Clinical course and outcomes of critically ill patients with SARS-CoV-2 pneumonia in Wuhan, China: a single-centered, retrospective, observational study. Lancet Res Med 8:475-481. https://doi.org/10.1016/S2213 $-2600(20) 30079-5$

5. Rajagopal K, Keller SP, Akkanti B, et al. (2020). Advanced pulmonary and cardiac support of COVID-19 patients: emerging 
recommendations from ASAIO - a living working document. circ: Heart Failure 13

6. Li X, Guo Z, Li B, et al. (2020). Extracorporeal membrane oxygenation for coronavirus disease 2019 in Shanghai, China: ASAIO J, 66: 475-481

7. Marullo AG, Cavarretta E, Biondi-Zoccai G, et al. (2020). Extracorporeal membrane oxygenation for critically ill patients with coronavirus-associated disease 2019: an updated perspective of the European experience. Minerva Cardioangiol

8. Lodigiani C, Iapichino G, Carenzo L et al (2020) Venous and arterial thromboembolic complications in COVID-19 patients admitted to an academic hospital in Milan, Italy. Thromb Res 191:9-14. https://doi.org/10.1016/j.thromres.2020.04.024

9. Helms J, Tacquard C, Severac F et al (2020) High risk of thrombosis in patients with severe SARS-CoV-2 infection: a multicenter prospective cohort study. Intensive Care Med. https://doi. org/10.1007/s00134-020-06062-x

10. Lubnow M, Philipp A, Foltan M et al (2014) Technical complications during veno-venous extracorporeal membrane oxygenation and their relevance predicting a system-exchange - retrospective analysis of 265 cases. PLoS ONE 9:e112316. https://doi. org/10.1371/journal.pone.0112316

11. Appelt H, Philipp A, Mueller T et al (2020) Factors associated with hemolysis during extracorporeal membrane oxygenation (ECMO) - comparison of VA- versus VV ECMO. PLoS ONE 15:e0227793. https://doi.org/10.1371/journal.pone.0227793

12. Hastings SM, Ku DN, Wagoner S et al (2017) Sources of circuit thrombosis in pediatric extracorporeal membrane oxygenation. ASAIO J 63:86-92. https://doi.org/10.1097/MAT.0000000000 000444

13. Lubnow M, Philipp A, Dornia C et al (2014) d-dimers as an early marker for oxygenator exchange in extracorporeal membrane oxygenation. J Crit Care 29:473.e1-473.e5. https://doi.org/10.1016/j. jcrc.2013.12.008

Publisher's Note Springer Nature remains neutral with regard to jurisdictional claims in published maps and institutional affiliations. 